\title{
Meister im Verbergen - Notfallmanagement beim Vogel
}

\author{
Cornelia Konicek
}

Im Notfall gilt es, die Symptome des Vogels zügig einzuschätzen und die Erkrankung unter Vermeidung von Stress, Schmerzen und Unwohlsein zu diagnostizieren. Erfahrung sowie ein schnelles, effizientes Arbeiten helfen Ihnen, den Patienten im Notfall gezielt zu stabilisieren.

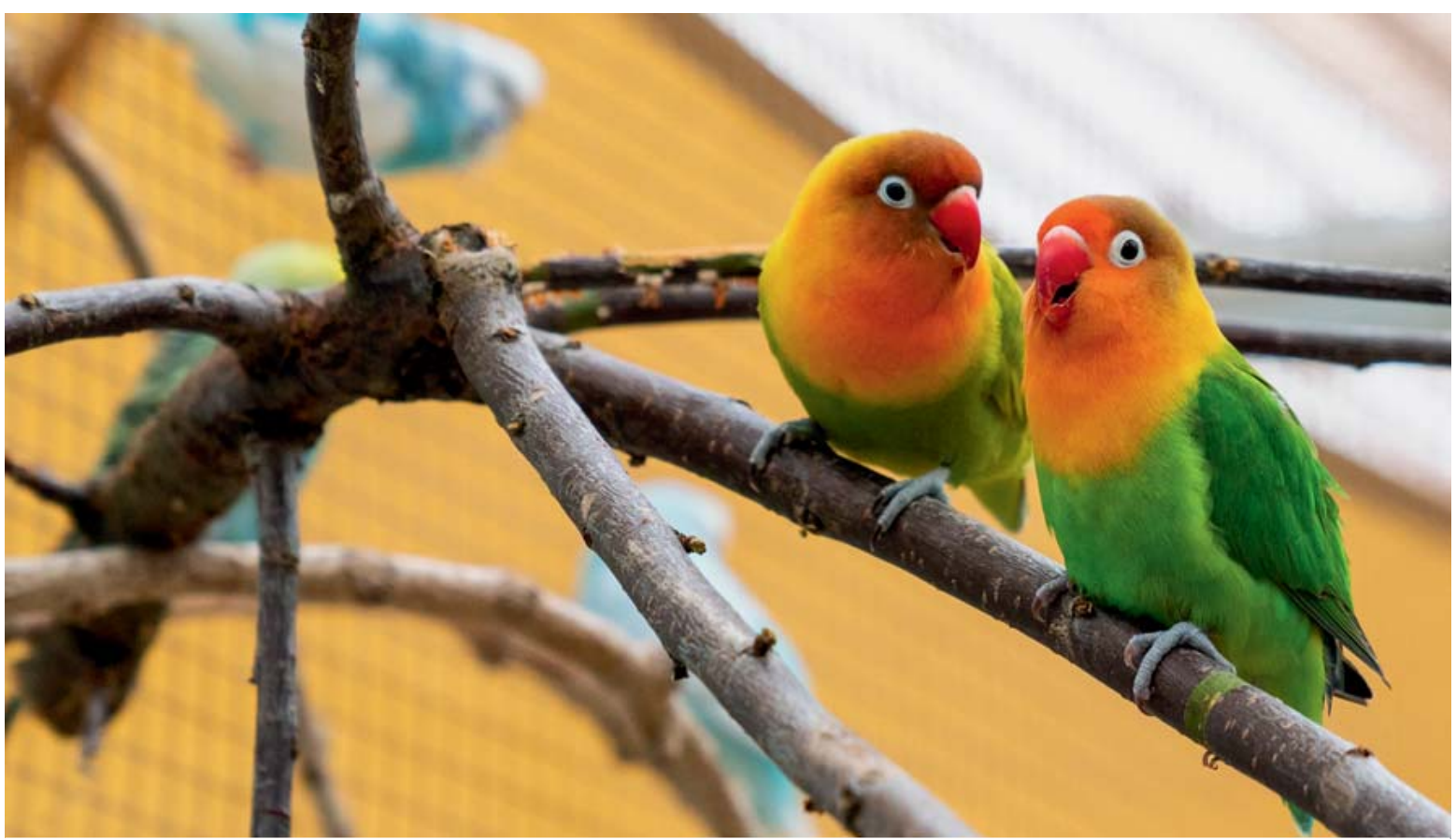

Quelle: Kirsten Oborny/Thieme Gruppe

Das Management von Notfällen und akuten Krankheitsverläufen gehört in der Exotensprechstunde nach wie vor zum Alltag. Auf der einen Seite ist der Vogel als Beutetier Meister im Verstecken von Krankheitssymptomen und zeigt klinische Symptome erst im weit fortgeschrittenen Krankheitsverlauf [1]. Auf der anderen Seite spielt die Präventivmedizin in den meisten Exotenpraxen/Kliniken noch eine weniger bedeutende Rolle ( $\bullet \mathbf{A b b}$. 1). Dabei wären einige Krankheitsursachen durch regelmäßige Vorsorgeuntersuchungen, entsprechendes Haltungsund Fütterungsmanagement sowie adäquate Beschäftigung und Training zu vermeiden. Eine gute Besitzerkommunikation und Compliance ist daher auch in der Notfallmedizin wichtig, um zumindest in der eigenen Klientel ein Umdenken zu bewirken.
Die Versorgung von einem Vogel-Notfall ist alleine aufgrund der Größe der Patienten, der unterschiedlichen Spezies mit ihren jeweiligen anatomischen und physiologischen Besonderheiten und der wenig vorhandenen evidenzbasierten Therapieempfehlungen eine Herausforderung. Dazu kommt, dass ein kranker Vogel den Transportstress und das Handling schlechter aushält als ein gesunder Artgenosse. Alleine die klinische Untersuchung bzw. das Handling können seinen Zustand akut verschlimmern und sogar zum Tod führen ( $\bullet$ Abb. 2) [2].

\section{Prinzipien der Notfallversorgung}

Wie in jeder Notfallsituation hat das ABC-Schema (Airways, Breathing, Circulation) Vorrang, bevor klinische Untersuchungen und Therapien angewendet werden kön- 
nen. Die Stabilisierung des Patienten hat oberste Priorität. Auch wenn einige diagnostische Tests eine bessere Einschätzung der Situation ermöglichen, kann es auch schnell zu viel werden. Wichtig ist ein rasches Einschätzen der Situation und so viel Stress, Schmerzen und Unwohlsein wie möglich zu vermeiden, was Erfahrung sowie ein schnelles und effizientes Arbeiten erfordert.

\section{Hyperoxygenierung ist ebenso schädlich wie Sauerstoffmangel}

Wichtig ist eine erste Beurteilung von Atemfrequenz, -qualität und -tiefe. Lunge und Luftsäcke können gut auskultiert werden. Knackende, feuchte, giemende Atemgeräusche sind auch beim Vogel pathologisch.

Schwer kranke Vögel werden sehr häufig zunächst mit Sauerstoff versorgt, was ihren Zustand oft verbessert und stabilisiert. Wissenschaftliche Daten dazu fehlen aber. Eine Hyperoxygenierung kann aber ebenso schädlich sein wie ein Sauerstoffmangel, das sollte im Hinterkopf behalten werden [3].

Merke

Bei Vögeln mit Atemproblemen und Zyanose sollte Sauerstoff verabreicht werden.

Wenn der Patient selbstständig atmet und ansprechbar ist, kann Sauerstoff direkt über eine Maske zugeführt werden, sofern das ohne großen Stress von dem Vogel toleriert wird. In den meisten Fällen ist es aber besser, ihn in eine sauerstoffreiche Umgebung zu setzen (Sauerstoffbox oder Sauerstoffkäfig). Dafür kann eine einfache Plastikbox mit Sauerstoff angereichert werden und der Vogel dort hineingesetzt werden ( $\vee$ Abb. 3).

\section{Bei Atemstillstand muss sofort intubiert und beatmet werden}

\section{Intubation}

Die Glottis liegt beim Vogel direkt hinter dem Zungengrund ( $>$ Abb. 4). Es gibt keine Epiglottis, was die Intubation leichter macht. Schwieriger ist es bei kleinen Papageienarten aufgrund der voluminösen Zunge und der kleinen Schnabelöffnung. Am leichtesten ist es, wenn man die Zunge mit einer atraumatischen Pinzette nach vorne zieht, dann findet man sehr schnell die dahinterliegende Öffnung zur Trachea. Achtung, die meisten Arten haben geschlossene Trachealspangen, daher dürfen nur Tuben ohne Cuff verwendet werden bzw. die Tuben nicht gecufft werden, da sonst schwerwiegende Schäden an der Trachea entstehen können.

\section{Katheterisierung des Luftsacksystems}

Das Luftsacksystem ( $>$ Abb. 5) der Vögel macht es möglich, die oberen Atemwege zu übergehen. Dazu kann ein Katheter in den kaudalen abdominalen oder kaudalen thorakalen Luftsack gesetzt werden. Dieser Eingriff ist

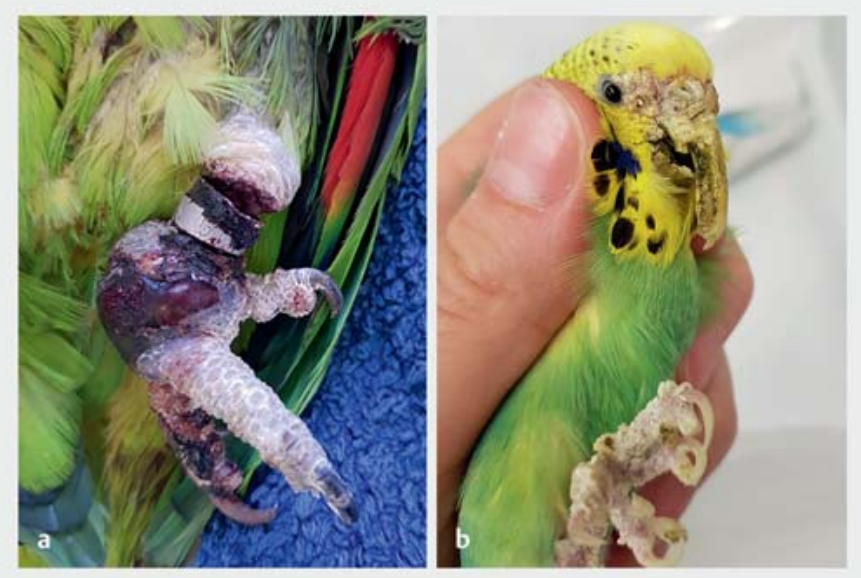

- Abb. 1 Manche „Notfälle“ könnten bei regelmäßiger Allgemeinuntersuchung und Beratung im Vorfeld verhindert werden. a Eingewachsener Fußring bei einer Amazone. b Hochgradige Manifestation von Räudemilben bei einem Wellensittich mit bereits übermäßig lang gewachsenem Oberschnabel. Quelle: Cornelia Konicek

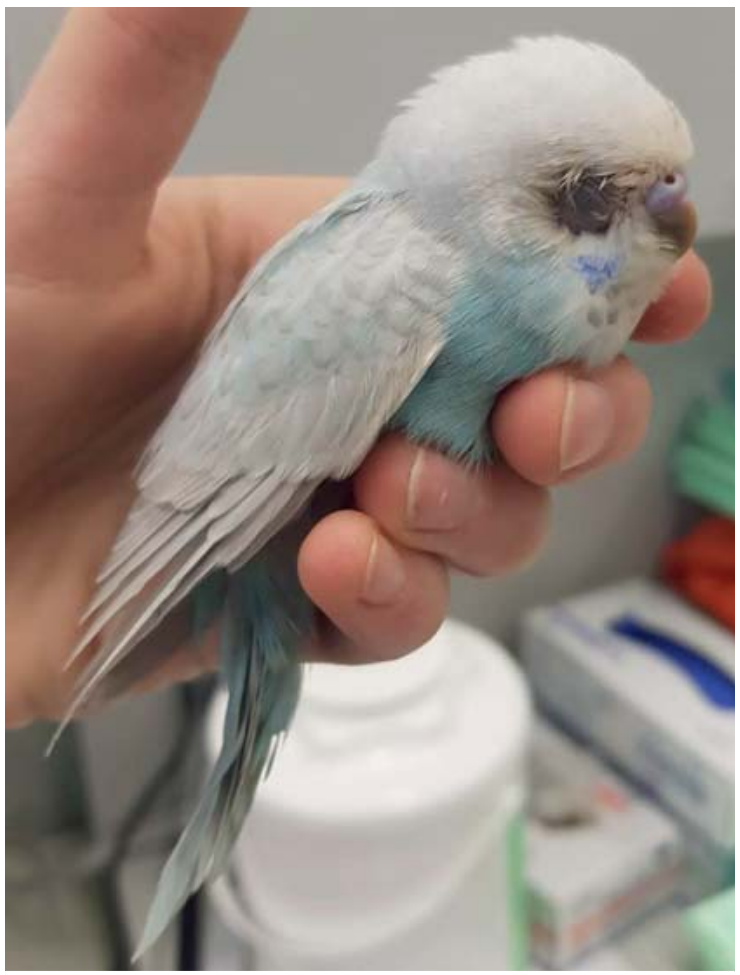

- Abb. 2 Vögel mit sehr schlechtem Allgemeinzustand, die kaum mehr Abwehrbewegungen zeigen, sollten so schnell und vorsichtig wie möglich untersucht werden. Ist der Allgemeinzustand bereits so stark vermindert, kann jeder weitere Stress zum vorzeitigen Tod führen. Quelle: Cornelia Konicek 


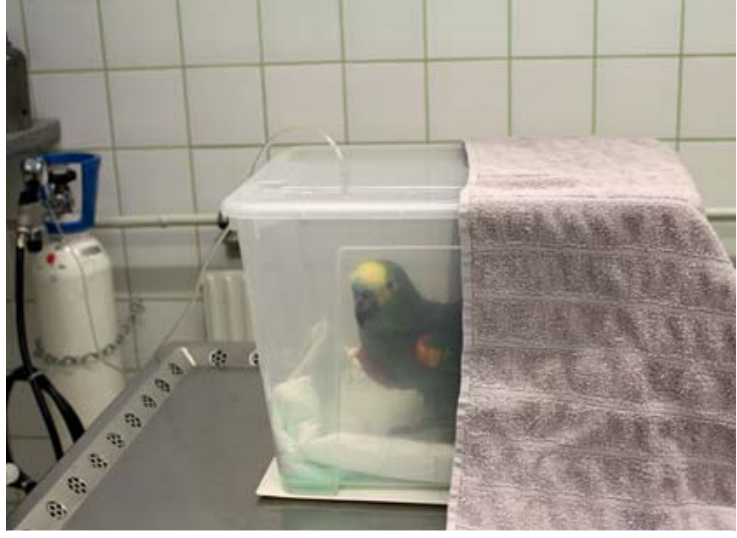

- Abb. 3 Plastikboxen mit Luftlöchern können im Notfall mit Sauerstoff angereichert werden und damit als „Sauerstoffboxen“ fungieren. Weich ausgepolstert eignen sich die Boxen auch gut für Vögel mit Krampfanfällen. Quelle: Cornelia Konicek

vor allem bei Obstruktionen der oberen Atemwege lebensrettend, kann aber auch bei kleinen Vogelarten zum Erhalt der Luftzirkulation eingesetzt werden, wenn eine Intubation aufgrund der Größe nicht möglich ist.

Dafür wird der Vogel bevorzugt in die rechte Seitenlage gebracht. Die Haut hinter der letzten Rippe und vor der Oberschenkelmuskulatur wird aseptisch vorbereitet ( $\bullet$ Abb. 6). Nach einem Hautschnitt mit einem Skalpell oder einer Injektionsnadel erfolgt ein stumpfer Zugang mit einer schmalen Péan-Klemme in die Leibeshöhle. Über den Zugang kann dann ein Katheter, der in etwa denselben Durchmesser wie die Trachea hat, in den kaudalen Luftsack eingesetzt werden. Der Katheter wird auf eine Länge gekürzt, die angenehm für den Vogel erscheint und wie ein Harnkatheter an der Haut fixiert. Über den Katheter kann direkt Sauerstoff eingeleitet werden, und der Vogel kann auch manuell beatmet werden. Bei sehr kleinen Patienten ist es am schnellsten und effektivsten, einen Venenkatheter in den Luftsack zu setzen (20-24 G) [4].

\section{Cave}

Achtung, Haut und Körperwand sind beim Vogel nur wenige Millimeter dick. Eine iatrogene Verletzung von inneren Organen mit dem Stilett ist bei einer Katheterisierung des Luftsacksystems möglich.

Sobald man den Venenkatheter in den Luftsack eingebracht hat, kann man das Stilett ziehen und den Zugang fixieren.

Der richtige Sitz kann adspektorisch gut beurteilt werden, die ausströmende warme Luft beschlägt den Katheter. Man bemerkt zudem bei manueller Ventilation schnell, ob Luftsäcke und Lunge oder nur die Haut venti-

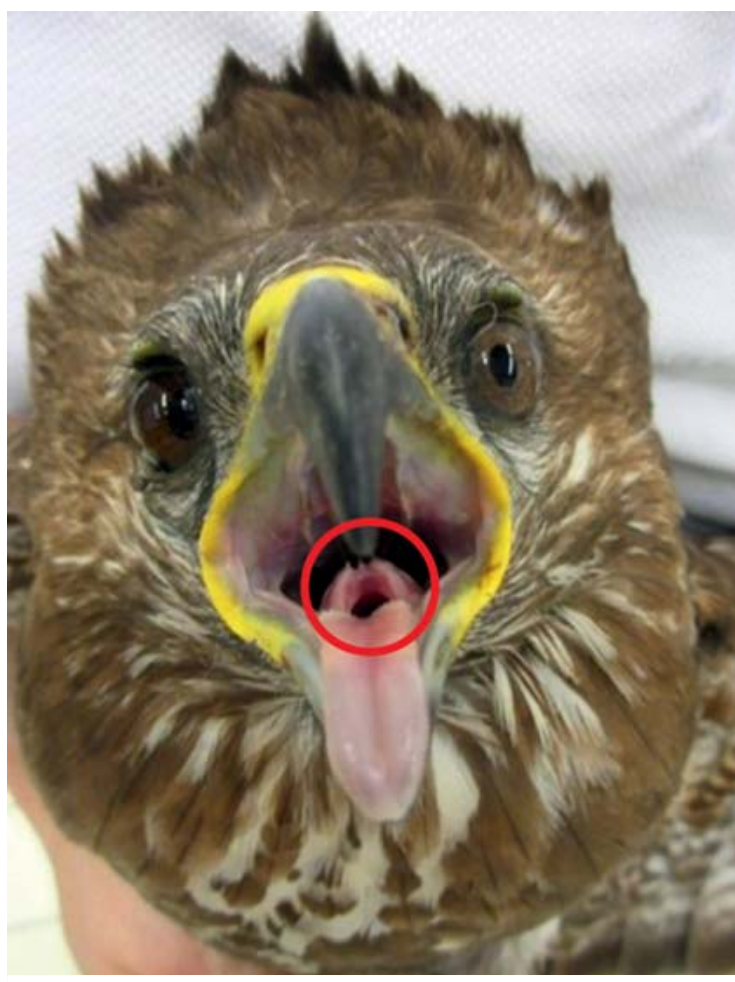

- Abb. 4 Die Glottis liegt beim Vogel direkt hinter dem Zungengrund, es gibt keine Epiglottis und keine Stimmbänder. Quelle: Cornelia Konicek

liert werden. Ebenso kann der korrekte Sitz mittels Kapnografen durch die Messung des exspiratorischen $\mathrm{CO}_{2}$ Werts kontrolliert werden. Es gibt keine genauen Daten zur notwendigen Zirkulationsrate. Empfehlungen gehen je nach Größe von 10-50 Atemzügen/Minute aus.

\section{Eine zügige kardiopulmonale Reanimation ist erforderlich}

Für die Beurteilung des Kreislaufs ist es hilfreich, die Schleimhautfarbe der Konjunktiven, wenn einsehbar der Schnabelhöhle und der Kloake zu beurteilen.

Bei verminderter Durchblutung zeigen die Tiere:

- einen verminderten Allgemeinzustand

- anämische Schleimhäute

- eine verlängerte Füllungszeit der Gefäße

- einen schwachen Puls

- Tachykardien

Bei einem kompletten Herz-Kreislauf-Stillstand kann eine kardiopulmonale Wiederbelebung versucht werden. Die Erfolgsrate ist allerdings gering. Eine reale Chance hat man nur bei sehr raschem Eingreifen, daher sollten ein Notfallset, Medikamente und ein Flowchart (Kasten) mit Dosierungen immer greifbar sein. 


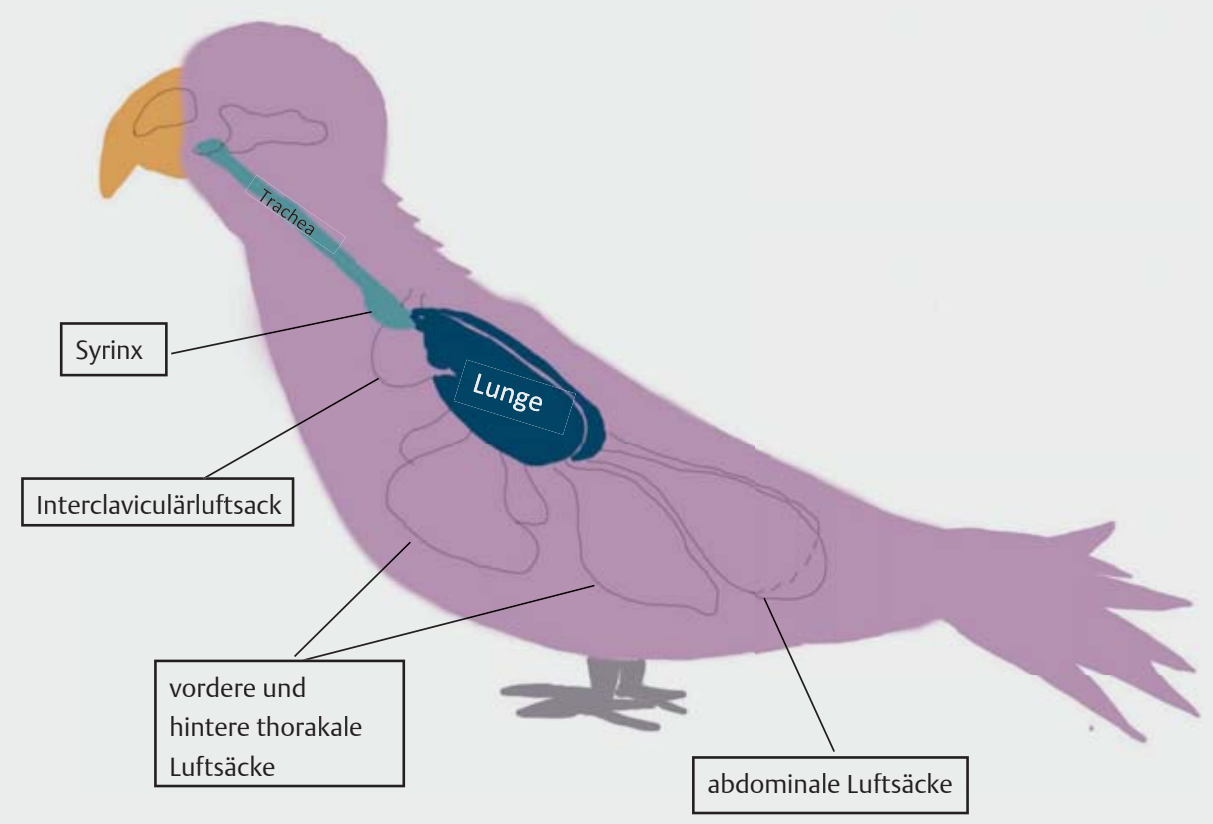

a

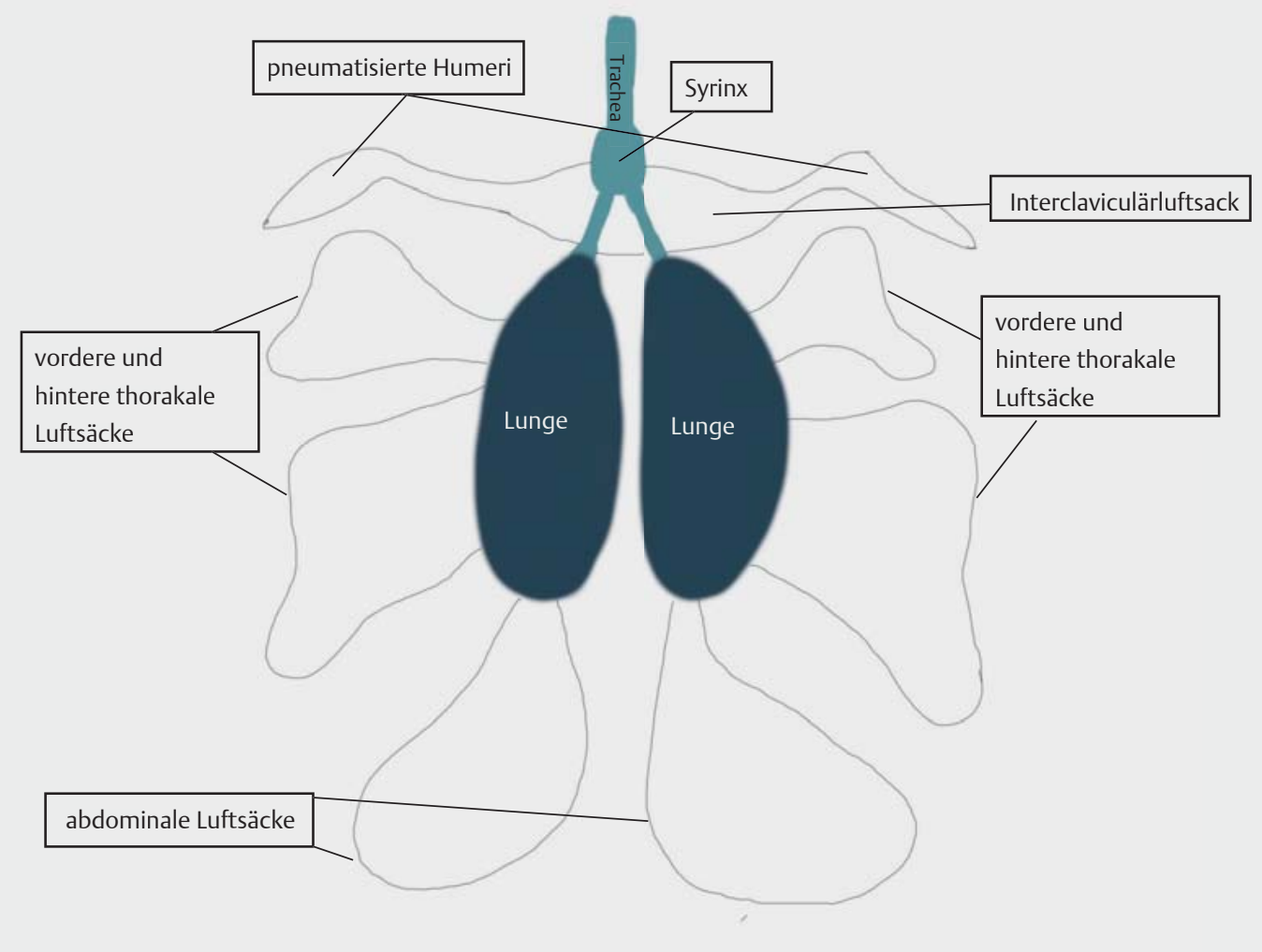

- Abb. 5 Schematische Abbildung der Lunge und Luftsäcke. Aufgrund des permanenten Gasaustauschs bei Inspiration und Exspiration können Vögel über einen Zugang in die hinteren Luftsäcke gut mit Sauerstoff versorgt und auch beatmet werden. Quelle: Cornelia Konicek 


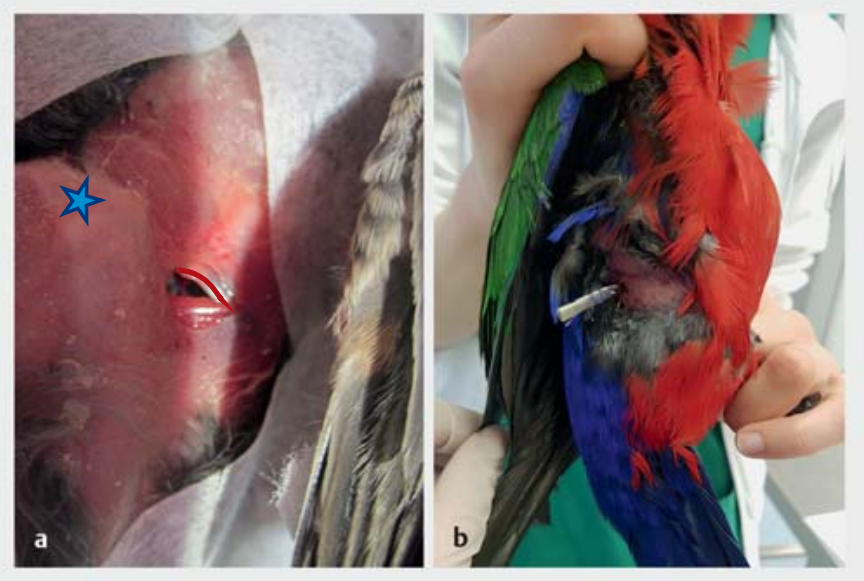

- Abb. 6 a Zugang für einen Luftsackkatheter (rote Markierung: letzte Rippe, Stern: Knie) bei einem Nymphensittich (Nymphicus hollandicus). Beim Setzen des Katheters muss die Hinterextremität nach kaudal gezogen werden, da sonst der Oberschenkel und die Muskulatur den Zugang versperren. b Ein mit „Chinese-finger-trap“-Naht fixierter Luftsackkatheter bei einem Amboina-Königssittich (Alisterus amboinensis) mit Aspergillose. Quelle: Cornelia Konicek

\section{NOTFALLPLAN}

Notfallplan für Vögel mit Atemstillstand/Herz-Kreislauf-Stillstand nach Lichtenberger und Lennox $[1,5]$ :

\section{Atemstillstand:}

- wenn unter Narkose: Narkosegas stoppen/Medikamente antagonisieren

- setzen eines Endotrachealtubus und beatmen mit $100 \%$ Sauerstoff

- bei Obstruktion der oberen Atemwege oder sehr kleinen Patienten: setzen eines Luftsackkatheters

- Doxapram 1-2 mg/kg i. m./i.v./i.o.

Bradykardie: Atropin $0,02 \mathrm{mg} / \mathrm{kg}$ i. v./i. o.

\section{Herz-Kreislauf-Stillstand:}

- Herzmassage 60-120/min (Effektivität ist fraglich)

- Epinephrin (Adrenalin) 0,01 mg/kg i.v./i. o.; $0,02 \mathrm{mg} / \mathrm{kg}$ endotracheal

Flüssigkeitsverluste über intravenösen oder intraossären Zugang ausgleichen

Kontrolle der inneren Körpertemperatur - bei Bedarf korrigieren

Diagnostik einleiten

Grundursache behandeln
Sobald der Vogel beatmet wird, kann bei komplettem Herzstillstand eine Brustkorbmassage durchgeführt werden, wobei 60-120 Kompressionen/Minute empfohlen werden. Da das Herz aber komplett von Rippen und Sternum eingeschlossen ist, ist es fraglich, ob eine Herzmassage durch Druck auf den Brustkorb beim Vogel auch wirklich möglich ist. Medikamentell kann bei Asystolie Epinephrin (Adrenalin) intrakardial gegeben werden; endotracheal oder intravenös sowie intraossär ist ebenfalls möglich.

Merke

Bei manueller Brustkorbmassage ist die intrakardiale Applikation der Medikamente aufgrund der fraglichen peripheren Durchblutung zu bevorzugen.

\section{Aufarbeitung eines Falles}

\section{Gezielte Anamnese und Adspektion bei stabilen Patienten}

Wenn Atmung und Kreislauf soweit stabil erscheinen, sollte eine gezielte Anamnese erhoben werden.

Wurde kein offensichtliches Trauma (Anflugtrauma, Bissverletzung etc.) beobachtet, sollten zunächst wichtige anamnestische Daten erfragt werden:

- Alter ( $\triangleright$ Tab. 1)

- Geschlecht inklusive Reproduktionsstatus (v.a. bei weiblichen Tieren)

- Neuzugänge im Bestand/Kontakt mit Artgenossen

- vorherige Erkrankungen bei diesem Patienten bzw. im Bestand

- kurze Beschreibung der Haltung und Fütterung

- Frage nach Freiflug

- Aufnahme von Fremdmaterial möglich? (v. a. metallene Gegenstände, verzinkte Gitter, Glöckchen, Schmuck, Lampen, Batterien, Vorhangbeschwerer)

- Exposition zu reizenden Dämpfen wie Rauch, Raumdüfte, Desinfektionsmittel, Teflondämpfe, Backrohrdämpfe oder Tabakrauch möglich?

- Letzte Futteraufnahme?

- Normaler Harn- und Kotabsatz (> Abb. 7)? Wurden Veränderungen beobachtet?

Stabile Vögel sollten in ihrem Käfig zunächst aus einiger Entfernung untersucht werden:

- Reaktion auf die fremde Umgebung: Sitzt der Vogel auf der Stange oder auf dem Boden?

- Atemfrequenz/Hinweise auf Atemnot (Schwanzatmung, abgespreizte Flügel, Atmung mit offenem Schnabel/Atemgeräusche, Backenblasen): Schon die Atemfrequenz kann erste Hinweise geben. Vögel mit weitgestelltem Stand, abgestreckten Flügeln und sehr tiefen Atemzügen haben oft Erkrankungen der kleinen Atemwege. Hingegen können kurze oberflächliche Atemzüge oftmals bei Raumforderungen der Leibes- 
- Tab. 1 Häufig vorgestellte Vogelarten, ihre Lebenserwartung in Gefangenschaft sowie ihr durchschnittliches Körpergewicht.

\begin{tabular}{|l|c|c|}
\hline Vogelart & Lebenserwartung (Jahre) & durchschnittliches Gewicht (Gramm) \\
\hline Kanarienvogel & $6-10$ & 20 \\
\hline Wellensittich & $5-10$ & 38 \\
\hline Nymphensittich & $10-25$ & 90 \\
\hline Graupapagei & $40-60$ & 410 \\
\hline Gelbbrustara & $30-40$ & 1000 \\
\hline
\end{tabular}
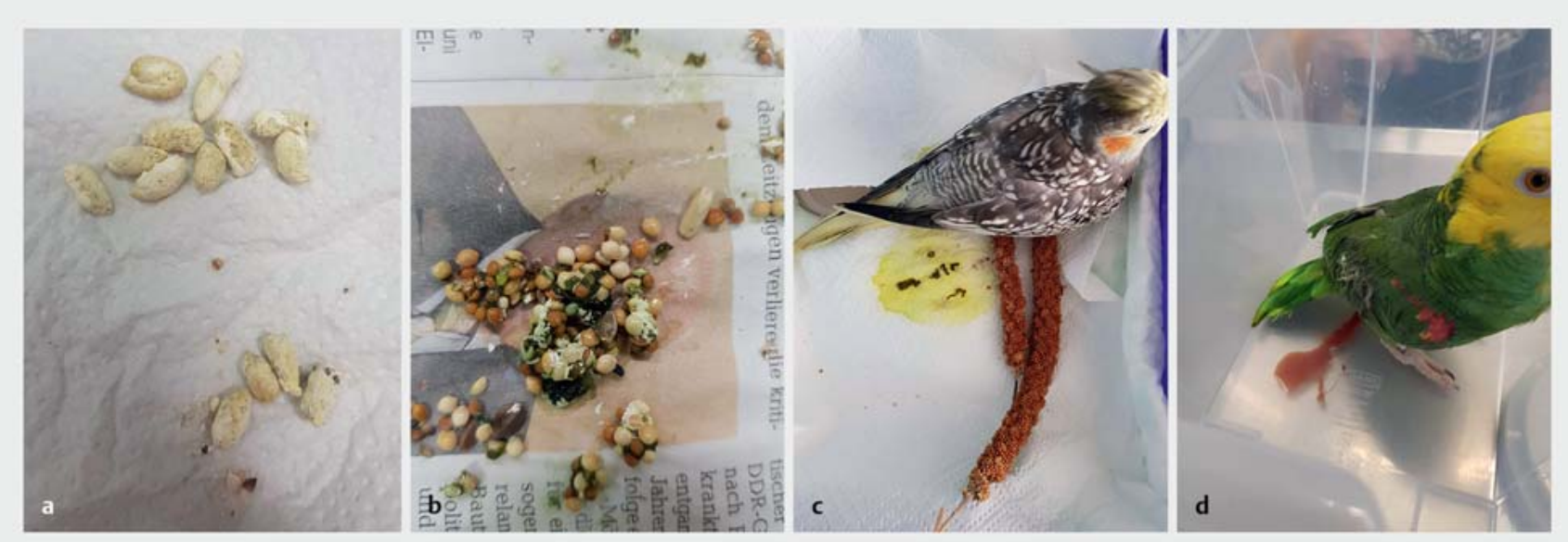

- Abb. 7 Die adspektorische Beurteilung von Kot und Harn kann bereits sehr aufschlussreich sein. a Dieser Kot eines Wellensittichs ist voluminös und weiß verfärbt, was typisch für eine Pankreasinsuffizienz ist. b Dieser Vogel scheidet hochgradig unverdaute Körner aus. Es sollte genau untersucht werden, ob die Körner wirklich mit dem Kot ausgeschieden wurden oder im Nachhinein auf den Kot gefallen sein könnten. c Dieser Nymphensittich (Nymphicus hollandicus) hat eine stark gelb verfärbte Harnsäure, was Hinweise auf eine Lebererkrankung geben kann. $\mathbf{d}$ Diese Doppelgelbkopfamazone (Amazona oratrix) scheidet blutigen Harn aus. In diesem Fall war eine Zinkintoxikation die Ursache. Quelle: Cornelia Konicek

höhle (Flüssigkeitsansammlungen, Tumore, Eier etc.) auftreten.

- Zeigt der Patient neurologische Symptome wie Opisthotonus, im Kreis laufen, Tremor, Krämpfe oder ungewöhnliche Vokalisation?

- Zudem sollte die Umgebung abgesucht werden: Gibt es Hinweise auf Erbrochenes, Kot- und Harnveränderungen? Wurden Kot und Harn überhaupt abgesetzt?

\section{Eine priorisierte klinische Untersuchung ist erforderlich}

Die klinische Untersuchung von Notfallpatienten muss schnell und nach Prioritäten erfolgen. Mithilfe der Anamnese und der Adspektion kann in den meisten Fällen bereits abgeschätzt werden, welche Maßnahmen/Diagnostik in diesem Fall wichtig und möglich wären und welche Medikamente der Patient brauchen könnte. Das reduziert die Zeit des notwendigen Handlings und erhöht damit die Überlebenschancen. In jedem Fall sollten bereits alle notwendigen Utensilien [6] und Medikamente sowie eine angewärmte Infusion vorbereitet werden. Wichtig sind Sauerstoff, hochprozentige Glukoselösung oder Traubenzuckerlösung zur oralen Eingabe sowie entsprechende Notfallmedikamente ( $\bullet$ Tab. 2).

Um die Aufregung beim Einfangen des Patienten zu minimieren, kann es hilfreich sein, den Raum etwas abzudunkeln. Bei Vögeln, die während des Handlings sehr gestresst erscheinen, empfiehlt sich eine leichte Sedierung mit Midazolam $(0,25-0,5 \mathrm{mg} / \mathrm{kg})$ und Butorphanol (1$2 \mathrm{mg} / \mathrm{kg}$ ) i.m. [5].

Vögel, die sehr schwach erscheinen, sollten zunächst weiter stabilisiert werden. Oftmals hilft zunächst eine warme subkutane Infusion (bei sehr schwachen Tieren kann selbst das Legen von intravenösen Zugängen oder intraossären Kathetern zu invasiv sein und zum Tod führen). Zusätzlich kann Glukoselösung oral verabreicht sowie Sauerstoff in den Käfig eingeleitet bzw. der Vogel in eine Sauerstoffbox umgesetzt werden. Danach sollte man den Patienten an einem ruhigen, abgedunkelten 
- Tab. 2 Wichtige Notfallmedikamente mit Dosierungsvorschlägen [2, 7].

\begin{tabular}{|c|c|c|c|}
\hline Wirkstoff & Dosis & Indikation & Anmerkungen \\
\hline Atipamazol & $0,1-0,2 \mathrm{mg} / \mathrm{kg}$ i.m./i.v. & $\begin{array}{l}\alpha 2 \text {-Adrenozeptor-Antago- } \\
\text { nist }\end{array}$ & - \\
\hline Flumazenil & $\begin{array}{l}0,02-0,05 \mathrm{mg} / \mathrm{kg} \text { i. m./i.v. } \\
0,05-0,25 \mathrm{mg} / \mathrm{kg} \text { intranasal }\end{array}$ & Benzodiazepin-Antagonist & - \\
\hline Atropin & $\begin{array}{l}0,02-0,5 \mathrm{mg} / \mathrm{kg} \text { i. m./i.v./i. o./endo- } \\
\text { tracheal/intrakardial }\end{array}$ & $\begin{array}{l}\text { Bradykardie, kardiopulmo- } \\
\text { nale Reanimation }\end{array}$ & - \\
\hline Doxapram & $\begin{array}{l}\text { 1-20 mg/kg i. m./i.v./i. o./endo- } \\
\text { tracheal/intrakardial }\end{array}$ & $\begin{array}{l}\text { Atemstillstand, kardiopul- } \\
\text { monale Reanimation }\end{array}$ & Wirkung fraglich \\
\hline $\begin{array}{l}\text { Epinephrin } \\
\text { (Adrenalin) } \\
(1: 1000)\end{array}$ & $\begin{array}{l}\text { 0,5-1 ml/kg i. m./i. v./i. o./endo- } \\
\text { tracheal/intrakardial }\end{array}$ & $\begin{array}{l}\text { Bradykardie, kardiopulmo- } \\
\text { nale Reanimation }\end{array}$ & - \\
\hline Infusionen & $\begin{array}{l}\text { 10-25 ml/kg i. v./i. o./als Bolus über } \\
\text { 5-7 Minuten } \\
\text { Erhaltungsbedarf: } 50-90 \mathrm{ml} / \mathrm{kg} / \text { Tag } \\
\text { s. c./i. m./i. v./i. o. }\end{array}$ & $\begin{array}{l}\text { Dehydratation, Hypo- } \\
\text { volämie }\end{array}$ & - \\
\hline Hetastarch (HES) & $\begin{array}{l}10-15 \mathrm{ml} / \mathrm{kg} \text { langsam i.v./i. o. } \\
\text { alle } 8 \text { Stunden }\end{array}$ & $\begin{array}{l}\text { Hypoproteinämie, Hypo- } \\
\text { volämie }\end{array}$ & 1-4 Anwendungen \\
\hline Mannitol & 0,2-2 mg/kg langsam i. v./i. o. & Hirnödem & nicht bei Hirnblutungen \\
\hline Kalziumglukonat & 50-100 mg/kg i.m./langsam i.v. & $\begin{array}{l}\text { Hypokalzämie, Hyper- } \\
\text { kaliämie }\end{array}$ & $\begin{array}{l}\text { verdünnt auf maximal } \\
50 \mathrm{mg} / \mathrm{ml} \text { anwenden }\end{array}$ \\
\hline Magnesiumsulfat & 20 mg/kg einmalig i. m. & Hypomagnesiämie & v.a. bei Graupapageien \\
\hline \multirow[t]{2}{*}{ Terbutalin } & 0,01 mg/kg p. o./i.m. alle 6 Stunden & $\begin{array}{l}\text { Psittaziden; } \alpha 2 \text {-selektiver } \\
\text { Bronchodilatator }\end{array}$ & \multirow[t]{2}{*}{ - } \\
\hline & $0,1 \mathrm{mg} / \mathrm{kg}$ p. o. alle $12-24$ Stunden & $\begin{array}{l}\text { Aras, Amazonen; Broncho- } \\
\text { dilatator; Pneumonie, ob- } \\
\text { struktive pulmonale Erkran- } \\
\text { kungen }\end{array}$ & \\
\hline
\end{tabular}

und warmen Ort zur Ruhe kommen lassen. Ungeeignet ist ein hochfrequentierter Raum oder eine Station mit Katzen und Hunden. Nach etwa 1 Stunde kann dann ein neuer Versuch gestartet werden, den Vogel genauer zu untersuchen.

\section{Die klinische Untersuchung in Etappen kann empfehlenswert sein}

Bei der klinischen Untersuchung sollte in allen Fällen der Ernährungszustand (Palpation der Brustmuskulatur) beurteilt werden, eine Palpation der Leibeshöhle erfolgen sowie der Füllungszustand des Kropfes untersucht werden. Die Leibeshöhle ist in der Regel schmal. Meist kann nur die Unterseite des Muskelmagens ertastet werden. Der Kropf kann leer oder mit Futter gefüllt sein.

Zu den möglichen tastbaren Abweichungen der Leibeshöhle zählen:

- deutliche Schwellung

- Flüssigkeitsansammlungen

- Tumore

- kaudoventrale Verdrängungen des Muskelmagens
- Vortreten des kaudalen Leberrands über das Sternum hinaus

- Eier

Zu den pathologischen Veränderungen des Kropfes gehören:

- eine verdickte Schleimhaut

- Flüssigkeits- oder Gasansammlungen

- Fremdkörper

- Tumore

Wenn der Vogel weiterhin stabil genug erscheint, sollte eine genaue klinische Untersuchung durchgeführt werden. Dazu gehört unter anderem der Hydrierungsstatus, der leider klinisch nicht so gut evaluierbar wie bei Kleintieren ist.

Zeichen einer starken Dehydration sind:

- trockene Schleimhäute

- Exophthalmus

- stehende Hautfalten an den oberen Augenlidern

- verzögerte Füllungszeit der Gefäße (z. B. V. basilica) 
- Tab. 3 Möglichkeiten zur klinischen Abschätzung des Dehydratationsgrads mit Empfehlungen zur Flüssigkeitssubstitution nach Riley und Barron [9].

\begin{tabular}{|l|l|l|}
\hline Dehydratationslevel & Klinik & Flüssigkeitssubstitution \\
\hline $5-10 \%$ & - verminderte Hautelastizität & - subkutan evtl. ausreichend \\
& - verzögerte Kapillarfüllungszeit & - bei ausbleibender klinischer Besserung intra- \\
& - gering klebrige Schleimhäute & venösen oder intraossären Zugang wählen \\
\hline$>10 \%$ & - Haut in Falten & - intravenös/intraossär \\
& - aufgehobene Hautelastizität & - zum Ausgleich evtl. kolloidale Lösungen verwen- \\
& - visköser Speichel & den \\
\hline & - trockener Kot und Urate & \\
& - Lethargie & \\
& - kühle Extremitäten & \\
& - Schock & \\
\hline
\end{tabular}

Zusätzlich sollten noch die Nasenlöcher, soweit einsehbar die Schnabelhöhle inklusive der Choanenspalte, die Kloakenumgebung und die Haut (besonders unter den Flügeln) untersucht werden.

Auskultatorisch kann es Hinweise auf Lungen- oder Herzerkrankungen geben. Herzgeräusche, Unregelmäßigkeiten, erhöhte oder verringerte Herzrate, pathologische (giemende/knackende/feuchte) Atemgeräusche sind als pathologisch zu beurteilen.

Bei zentralnervösen Symptomen oder vorberichtlichem Schädel-Hirn-Trauma sollte auch immer eine neurologische Untersuchung inklusive Kopfnervenbeurteilung und Augenhintergrund erfolgen. Während des Handlings der Patienten sollte man darauf achten, ob sich ihr Zustand rasch verschlechtert. Treten plötzlich verstärkte Atemnot oder Krämpfe auf oder wird der Vogel immer weniger wehrhaft, muss die Untersuchung sofort abgebrochen bzw. pausiert werden. Wenn notwendig, müssen die Untersuchungsschritte und Probennahmen in Etappen durchgeführt werden.

\section{Stabilisierung und Erstversorgung des Patienten}

\section{Die Flüssigkeitstherapie richtet sich nach dem Grad der Dehydratation}

Bei Vögeln mit klinischen Symptomen einer Hypoperfusion oder hypovolämischem Schock ist eine Flüssigkeitssubstitution notwendig ( $\triangleright$ Tab. 3 ).

Intravenöse Katheter können in die Flügelvenen ( $\triangleright$ Abb. 8), die mediale Metatarsalvene oder auch in die Jugularvene gesetzt werden. Alternativ kann ein intraossärer Zugang in den proximalen Tibiotarsus, in die distale oder proximale Ulna gesetzt werden.

\section{Cave \\ Achtung, keine Flüssigkeitsgabe in die Humeri oder Femora, da diese pneumatisiert und somit mit dem Respirationstrakt verbunden sind.}

Man muss beachten, dass intraossäre Infusionen auch für den Vogel unangenehm schmerzhaft sein können, wie es in der Humanmedizin beschrieben ist. Bei intraossären Zugängen kann eine möglichst langsame Infusionsrate hilfreich sein, um es weniger unangenehm zu machen. Zusätzlich sollte beim Setzen des Zugangs die Applikationsstelle lokal mit Lidocain betäubt werden. Der Vogel kann, falls er bei Bewusstsein ist, in eine kurze Narkose gelegt werden.

Bei sehr schwachen Tieren ist eine erste subkutane Flüssigkeitssubstitution (50-100 ml/kg, > Abb. 9) sinnvoll, bevorzugt in die Inguinalregion.

Die Katheter müssen immer gut mit Tape fixiert und/ oder an die Haut genäht werden. Bitte nur „hautfreundliches" Tape benutzen, da bei Abnahme sonst Feder und Haut verletzt werden können. Bei Kathetern, die in den Flügel gesetzt werden, sollte der ganze Flügel mit einem Achterverband ruhiggestellt werden. Je nach Art und Allgemeinzustand können weitere Sicherungsmaßnahmen wie ein Halskragen ( $\triangleright$ Abb. 10) und ein entsprechender Schutz um den Infusionsschlauch (feste Plastikschläuche, z. B. Aquarienschläuche durch die die Infusionsleitung gezogen wird) notwendig sein.

\footnotetext{
Cave

Ein Vogel mit intravenösem Zugang sollte nie längere Zeit unbeaufsichtigt bleiben, da es beim Rausreißen des Zugangs zu fatalen Blutungen kommen kann.
} 


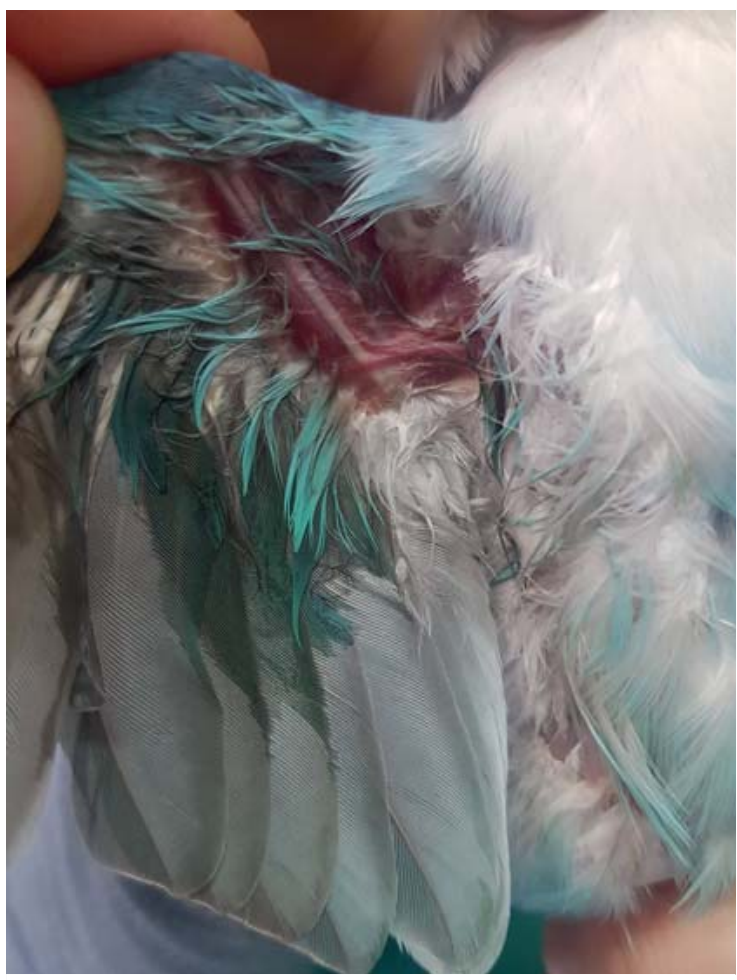

- Abb. 8 Aufzweigung der V. brachialis in die V. ulnaris/ V. basilica und V. radialis. Diese Gefäße eignen sich zur Blutprobenentnahme sowie für intravenöse Zugänge bei Vögeln ab ca. $100 \mathrm{~g}$ Körpergewicht. Quelle: Cornelia Konicek

\section{Welche Infusionslösungen bieten sich an?}

Bei hypovolämischem Schock hat sich die Gabe von kolloidaler Flüssigkeit (z.B. Hetastarch ${ }^{\circledR}$ ) bewährt. Dabei sind $10 \mathrm{ml} / \mathrm{kg}$ als Boli über 10 Minuten empfehlenswert, die so oft wiederholt werden können, bis sich der Patient stabilisiert hat. Ebenso können kristalloide Lösungen verwendet werden (30-60 ml/kg über 1 Stunde). Dabei gilt es, immer gut auf mögliche Symptome einer Hyperperfusion bzw. Paravasation zu achten. Wenn sich die Symptome der Hypoperfusion gebessert haben, kann auf eine Erhaltungsrate von $10 \mathrm{ml} / \mathrm{kg} / \mathrm{h}$ umgestellt werden.

Kristalloide Lösungen eignen sich gut als Rehydratationslösungen $(50 \mathrm{ml} / \mathrm{kg} / \mathrm{d}$ über 12-24 Stunden; bei HerzLungen-Erkrankungen über 24-36 Stunden).

\section{Vögel tolerieren Blutverluste besser als Säugetiere}

Obwohl Vögel akute und chronische Blutverluste besser tolerieren, gibt es Indikationen, in denen eine Bluttransfusion eine lebensrettende Maßnahme sein kann. Idealerweise sollte es Blut von derselben Spezies sein. Fremdspezies sind möglich, allerdings mit einer deutlich verkürzten Lebensdauer der Erythrozyten. Idealerweise sollte vor Transfusion eine Cross-Matching-Probe durchgeführt werden. Da Vögel aber keine vorgebildeten Anti-

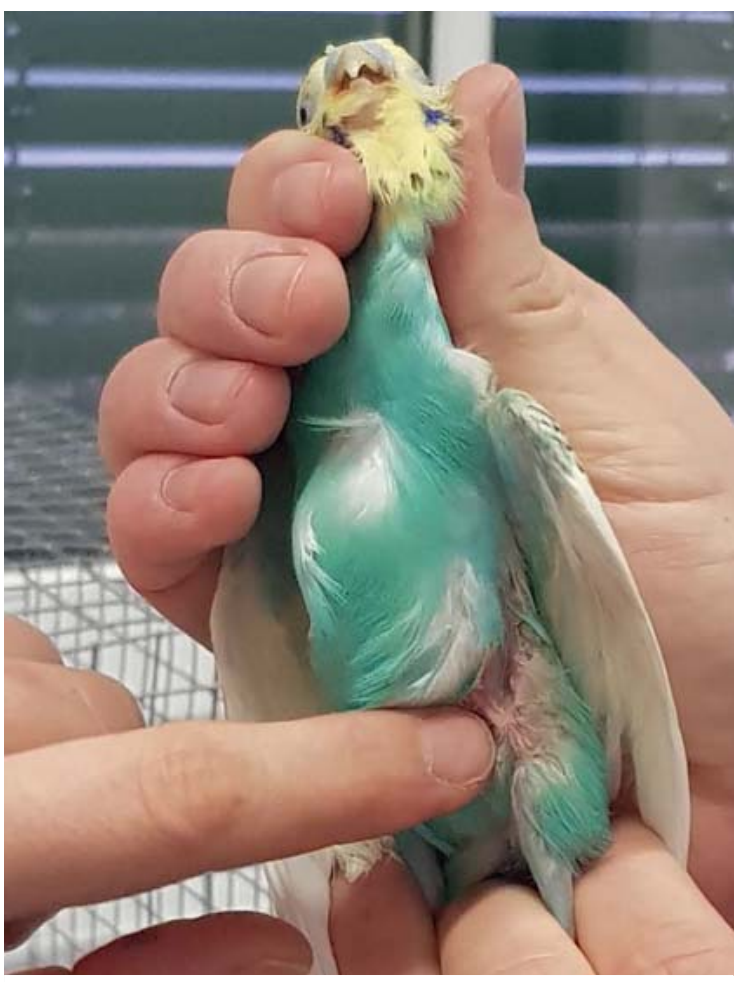

- Abb. 9 Eine gute Stelle für subkutane Infusionen ist die Inguinalregion bzw. die Kniefalte. Der Bereich ist bei einem Wellensittich (Melopsittacus undulatus) durch Anfeuchtung mit einem Desinfektionsmittel freigelegt. Quelle: Cornelia Konicek

körper für Blutgruppen aufweisen, ist die 1. heterogene Transfusion sicher [10].

Indikationen sind:

- starker Blutverlust (> 20\% des Blutvolumens)

- Hypoproteinämie

- Perfusionsdefizite

- Patienten mit chronischer Anämie, die operiert werden müssen

Kontraindikationen sind:

- Normovolämie

- Dehydratation

- geringe bis mittelschwere chronische Anämie

- Anämie in Kombination mit Dehydratation

- Anämie in Kombination mit Hypoproteinämie

\section{Blutdruckmessungen sind auch beim Vogel möglich}

Nicht invasive Messungen sind an der Flügelvene (V. basilica) und der medialen Metatarsalvene (V. metatarsalis superficialis plantaris) möglich. Allerdings weisen vergleichende Studien mit invasiver Blutdruckmessung deutliche Messunterschiede auf, daher sind die nicht invasiven Blutdruckmessungen mit großer Vorsicht zu interpretieren [11]. 


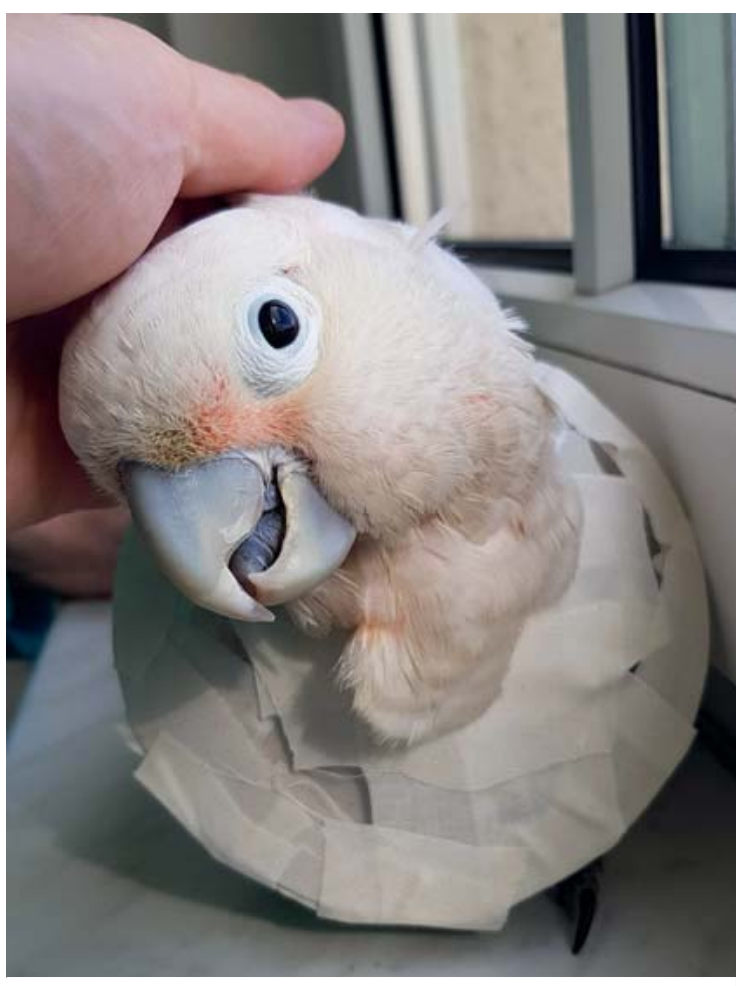

- Abb. 10 Nicht alle Patienten akzeptieren ihre Zugänge und Verbände. Bei manchen muss zusätzlich ein Halskragen angelegt werden. Quelle: Cornelia Konicek

\section{Vögel kühlen rasch aus}

Vor allem bei kleinen Patienten ist es sehr wichtig, auf die Umgebungstemperatur zu achten, da sie aufgrund der großen Körperoberfläche rasch auskühlen. Inkubatoren eignen sich zur Wärmetherapie generell besser als Wärmematten oder Wärmelampen. Wenn Wärmematten oder -lampen verwendet werden, muss der Vogel immer die Möglichkeit haben, der Wärmequelle auszuweichen. Für die meisten Arten sind Temperaturen zwischen $29-32{ }^{\circ} \mathrm{C}$ und eine Luftfeuchtigkeit von 50-60\% empfehlenswert.

\section{Nie auf einen vollen Kropf zwangsernähren}

Anorektische Tiere oder Tiere, die trotz Futteraufnahme ihr Gewicht nicht halten können, müssen zwangsernährt werden ( $\triangleright$ Abb. 11). Das geht in der Regel leicht über eine Knopfkanüle, die in den Kropf eingeführt wird. Einige entsprechende Produkte sind bereits am Markt für omnivore, carnivore und piscivore Spezies.

Es gilt:

- In etwa $20 \mathrm{ml} / \mathrm{kg}$ Futterbrei können pro Fütterung eingegeben werden.

- Bei Passerinen kann die Fütterung alle 2 Stunden und bei Psittaciden alle 3-8 Stunden erfolgen.

Wichtig ist, dass der Kropf sich vor der nächsten Fütterung immer entleert hat. Man sollte nie auf einen vollen Kropf füttern. Die Zwangsfütterung sollte immer die letz-

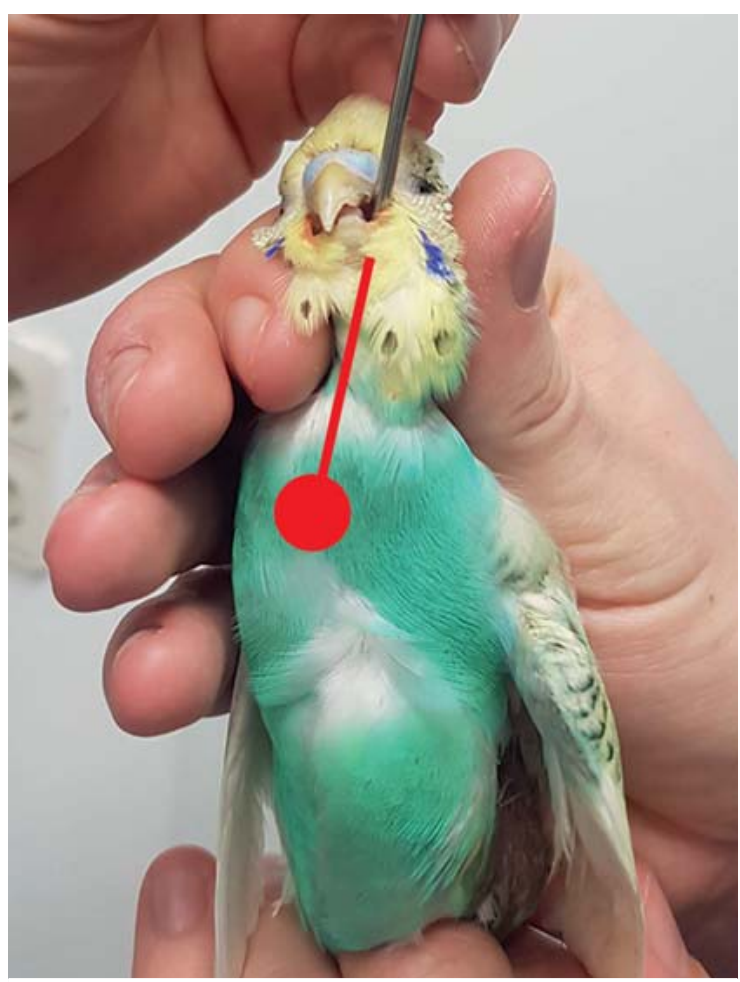

- Abb. 11 Setzen einer Kropfsonde bei einem Wellensittich (Melopsittacus undulatus). Quelle: Cornelia Konicek

te therapeutische Maßnahme sein, bevor der Vogel wieder in seinen Käfig gesetzt wird, um ein Regurgitieren zu vermeiden. Sollte der Vogel bereits während der Applikation regurgitieren, sollte sofort abgebrochen werden und der Vogel zurück auf den Käfigboden gesetzt werden, damit er den Brei nicht aspiriert und erstickt.

\section{Welche Analgesie bietet sich an?}

Ein gutes Schmerzmanagement sollte bei Vögeln immer Anwendung finden, sobald schmerzhafte Verletzungen/ Traumen bekannt sind, es klinische Zeichen gibt, die auf Schmerzen hinweisen und natürlich, wenn operative Eingriffe vorgenommen werden müssen [12].

Bisher wurde eine stärkere Ausprägung von k-Rezeptoren im Vergleich zu $\mu$-Rezeptoren nachgewiesen. Daher spielen к-Rezeptoren-Agonisten aktuell eine bedeutendere Rolle in der Schmerztherapie [13]. Butorphanol $1 \mathrm{mg} / \mathrm{kg}$ i. m. (0,5-2 mg/kg sind möglich) wird in den meisten Fällen empfohlen. Die Wirkdauer ist allerdings kurz, man geht von einer Analgesie von etwa 4 Stunden aus. Daher ist eine häufige Gabe notwendig ist. In Liposome gehülltes Butorphanol scheint vielversprechend eine längere Wirkdauer zu haben. Kürzlich wurde aber gezeigt, dass es Differenzen bei der Genexpression von $\mu$ - und к-Rezeptor-Agonisten bei verschiedenen Arten und auch Geweben gibt [14], sodass $\mu$-Rezeptor-Agonisten nicht generell außer Acht gelassen werden sollten. 
Bei weniger starken Schmerzen kann auch Tramadol (5$50 \mathrm{mg} / \mathrm{kg}$ i.m./p.o. alle 12 Stunden) gegeben werden; bei Neuralgien auch Gabapentin (10-30 mg/kg p. o. alle 8-12 Stunden) [15]. Ebenso können nicht steroidale Antiphlogistika (NSAID) verabreicht werden. Vor allem Meloxicam (1 mg/kg p.o./i. m./s. c. alle 12 Stunden) scheint sicher und wirksam. Vorsicht sollte dennoch bei dehydrierten Patienten geboten sein. Ebenso sind einige andere NSAID beschrieben und in Verwendung, jedoch mit unterschiedlicher Toxizität, je nach Dosis und Spezies.

\section{Kortikosteroide}

Die Gabe von Kortikosteroiden beim Vogel ist sehr umstritten. Hauptindikation sind Schädel-Hirn-Traumata, wobei das Trauma nicht länger als 24 Stunden zurückliegen sollte und die Gabe maximal ein 2. Mal wiederholt werden soll. Einige Praxen/Kliniken raten von der Kortikosteroid-Gabe komplett ab, da es keine wissenschaftlichen Daten gibt, die die Anwendung unterstützen. Sicher ist jedoch, dass es zu einer starken Immunsuppression kommt und sekundäre Infektionen begünstigt. Vor allem eine sekundär auftretende Schimmelpilzmykose sollte im Hinterkopf behalten und die Tiere im Zweifelsfall prophylaktisch mit Antimykotika behandelt werden [16].

\section{Nach der Stabilisierung folgt die diagnostische Aufarbeitung}

Ist der Patient stabilisiert, können weitere therapeutische Maßnahmen sowie eine notwendige Diagnostik durchgeführt werden.

Je nach Indikation und Stabilität des Patienten sind folgende Untersuchungen empfehlenswert [1]:

- Blutuntersuchung

- zumindest Hämatologie, Hämatokrit, Leukozytenzahl

- Blutchemie

- bei Verdacht auf Schwermetallintoxikation: Plasmaspiegel Blei und Zink (evtl. Kupfer)

- zytologische Untersuchungen

- Kropfspülprobe/feuchter Kropfabstrich nativ, Kot nativ

- gefärbte Abstriche von Rachen, Kropf, Kloake, Kot

- mikrobiologische Untersuchungen

- je nach Indikation Abstriche von Wunden, Nasenlöchern, Konjunktiven, Choane, Kropf, Kloake, Kot

- parasitologische Untersuchungen

- Rachenabstriche

- Kropfspülproben

- Kot

- bildgebende Diagnostik

- Röntgen

- Ultraschall: Leibeshöhle/Herz

- CT/MRT

\section{Fazit}

Für ein erfolgreiches Notfallmanagement beim Vogel ist eine rasche Einschätzung des Allgemeinzustands wichtig. Man sollte schon vor dem ersten Handling einen ungefähren Plan haben, welche Untersuchungen unmittelbar notwendig sind, damit auch alle erforderlichen Utensilien und Medikamente zur ersten Kreislaufstabilisierung griffbereit sind. Die direkte Untersuchung sollte möglichst ohne unnötigen Stress, Schmerz und so schnell wie möglich erfolgen. Eine gute Besitzercompliance ist auch in der Exotenmedizin wichtig. Vögel sind langjährige Familienmitglieder. Der Besitzer sollten daher im Vorfeld informiert werden, wie kritisch der Zustand erscheint und dass jedes Handling zu einem vorzeitigen Tod führen kann.

Korrespondenzadresse

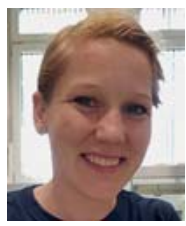

\section{Dr. Cornelia Konicek}

Veterinärmedizinische Universität Wien Klinik für Interne Medizin Kleintiere Service für Vögel und Reptilien Veterinärplatz 1

1210 Wien

Österreich

Cornelia.konicek@vetmeduni.ac.at

Literatur

[1] Bowles H, Lichtenberger M, Lennox A. Emergency and critical care of pet birds. Vet Clin North Am Exot Anim Pract 2007; 10: 345-394

[2] Stout JD. Common emergencies in pet birds. Vet Clin North Am Exot Anim Pract 2016; 19: 513-541

[3] Jenkins JR. Critical care of pet birds. Vet Clin North Am Exot Anim Pract 2016; 19: 501-512

[4] de Matos R, Morrisey JK. Emergency and critical care of small psittacines and passerines. Semin Avian Exotic Pet Med 2005; 14: $90-105$

[5] Lichtenberger M, Lennox A. Critical care. In: Speer B, ed. Current Therapy in Avian Medicine and Surgery. St. Louis, Missouri: Elsevier; 2016

[6] Hildreth CD. Preparing the small animal hospital for avian and exotic animal emergencies. Vet Clin North Am Exot Anim Pract 2016; 19: 325-345

[7] Carpenter JW, Hawkins MG, Barron H. Appendix 1: Table of common drugs and approximate doses. In: Speer B, ed. Current Therapy in Avian Medicine and Surgery. St. Louis, Missouri: Elsevier; 2016

[8] Costello MF. Principles of cardiopulmonary cerebral resuscitation in special species. Semin Avian Exotic Pet Med 2004; 13: 132-141

[9] Riley J, Barron H. Wildlife emergency and critical care. Vet Clin North Am Exot Anim Pract 2016; 19: 613-626

[10] Martinho F. Indications and techniques for blood transfusion in birds. J Exotic Pet Med 2009; 18: 112-116

[11] Comolli J, d'Ovidio D, Adami C et al. Technological advances in exotic pet anesthesia and analgesia. Vet Clin North Am Exot Anim Pract 2019; 22: 419-439 
[12] Lierz M, Korbel R. Anesthesia and analgesia in birds. J Exot Pet Medicine 2012; 21: 44-58

[13] Kubiak M. Avian analgesia. Comp Anim 2016; 21: 480-484

[14] Fousse SL, Golsen BM, Sanchez-Migallon Guzma D et al. Varying expression of mu and kappa opioid receptors in cockatiels (Nymphicus hollandicus) and domestic pigeons (Columba livia domestica). Front Genet 2020; 549558

[15] Baine K, Jones MP, Cox S et al. Pharmacokinetics of compounded intravenous and oral gabapentin in hispaniolan amazon parrots (Amazona ventralis). J Avian Med Surg 2015; 29 : $165-173$
[16] Verstappen FALM, Dorrestein GM. Aspergillosis in amazon parrots after corticosteroid therapy for smoke-inhalation injury. J Avian Med Surg 2005; 19: 138-141

Bibliografie

Kleintier konkret 2021; 24: 18-29

DOI $10.1055 / \mathrm{a}-1350-1634$

ISSN $1434-9132$

(C) 2021. Thieme. All rights reserved.

Georg Thieme Verlag KG, Rüdigerstraße 14, 70469 Stuttgart, Germany 